\title{
Association between chronological depressive changes and physical symptoms in postoperative pancreatic cancer patients
}

Naoko Sato ${ }^{1,5^{*}}$, Yoshimi Hasegawa ${ }^{2}$, Asami Saito ${ }^{1}$, Fuyuhiko Motoi ${ }^{3}$, Kyohei Ariake $^{3}$, Yu Katayose ${ }^{3}$, Kei Nakagawa ${ }^{3}$, Kei Kawaguchi ${ }^{3}$, Shin Fukudo ${ }^{4}$, Michiaki Unno ${ }^{3}$ and Fumiko Sato ${ }^{1}$

\begin{abstract}
Background: Pancreatic cancer (PC) has poorer prognosis and higher surgical invasiveness than many other cancers, with associated psychiatric symptoms including depression and anxiety. Perioperative depression has not been investigated in PC patients regarding surgical stress and relevant interventions.

Methods: We evaluated chronological depressive changes and subjective physical symptoms in surgically treated PC patients preoperatively and at 3 and 6 months postoperatively.

Enrolled patients undergoing pancreatic tumor surgery completed questionnaires based on the Self-Rating Depression Scale (SDS) and Functional Assessment of Cancer Therapy for Patients with Hepatobiliary Cancer (FACTHep) preoperatively, and at 3 and 6 months postoperatively. Responses were analyzed with JMP® Pro using oneway and two-way ANOVA, Spearman's rank correlation coefficient, and multiple regression analysis.

Results: Malignancy was diagnosed in 73 of 101 patients postoperatively; SDS score was significantly higher in these patients than in those with benign tumors at all timepoints: malignant/benign, 41.8/37.9 preoperatively $(p=0$. 004); $43.5 / 37.83$ months postoperatively $(p=0.006)$; and $42.9 / 37.76$ months postoperatively $(p=0.020)$. SDS scores were significantly higher in patients $<65$ years old with malignancy at 3 months than at 6 months postoperatively $(44.6 / 42.5, p=0.046)$ and in patients with malignancy who underwent pancreaticoduodenectomy at 3 months postoperatively than preoperatively $(43.4 / 41.1 ; p=0.028)$. SDS scores moderately correlated with 8 physical symptom-related FACT-Hep items 3 months postoperatively $(p<0.05)$, showing low-to-moderate correlation with 16 physical symptom-related FACT-Hep items at 6 months postoperatively $(p<0.05)$. Multiple regression analysis of FACT-Hep symptoms significantly correlated with SDS scores revealed the following significant variables: "lack of energy" ( $p<0.000)$ and "pain" ( $p=0.018)$ preoperatively $\left(R^{2}=0.43\right)$; "able to perform usual activities" $(p=0.031)$ and "lack of energy" $(p<0.000)$ at 3 months postoperatively $\left(R^{2}=0.51\right)$; and "stomach swelling or cramps" $(p=0.034)$ and "bowel control" ( $p=0.049)$ at 6 months postoperatively $\left(R^{2}=0.52\right)$.
\end{abstract}

Conclusions: PC patients experience persistently high levels of depression preoperatively through 6 months postoperatively, with associated subjective symptoms including pain and gastrointestinal symptoms.

Trial registration: UMIN Clinical Trials Registry 000009592, Registered 20 December 2012.

Keywords: Pancreatic cancer, Depression, Physical symptoms

\footnotetext{
* Correspondence: naoko-s@med.tohoku.ac.jp

'Department of Oncology Nursing, Tohoku University Graduate School of

Medicine, Sendai, Japan

${ }^{5}$ Department of Oncology Nursing, Tohoku University, 2-1 Seiryo-machi,

Aoba-ku, Sendai 980-8575, Japan

Full list of author information is available at the end of the article
}

(c) The Author(s). 2018 Open Access This article is distributed under the terms of the Creative Commons Attribution 4.0 International License (http://creativecommons.org/licenses/by/4.0/), which permits unrestricted use, distribution, and reproduction in any medium, provided you give appropriate credit to the original author(s) and the source, provide a link to the Creative Commons license, and indicate if changes were made. The Creative Commons Public Domain Dedication waiver (http://creativecommons.org/publicdomain/zero/1.0/) applies to the data made available in this article, unless otherwise stated. 


\section{Background}

Pancreatic cancer (PC) has a poorer prognosis than other cancers [1]. Although the 5-year survival rate in patients with localized cancer at the time of detection has increased to $30 \%$ following advances in medical science, more than $50 \%$ of PC patients have distant metastases when the cancer is diagnosed, and the overall survival rate for PC remains low, at 8\% [1]. Surgical resection is associated with a higher survival rate for PC [2] and is currently the sole treatment by which complete cure might be expected [3]. However, such treatment entails a high level of surgery-related stress and is associated with high rates of postoperative mortality and complications [4-7].

Pancreatic tumor (PT) resection causes high surgical stress regardless of whether the tumor is benign or malignant, and deterioration of quality of life (QOL) is a concern even when a benign tumor is diagnosed based on postoperative histology [5]. In light of concern over future malignant conversion, health professionals should consider a common approach to the physical and mental health of pancreatic resection patients facing surgical stress in the perioperative period, with no separate distinction made for those that receive a diagnosis of malignancy.

We reported on the extent of surgical stress in pancreatic resection and symptoms necessitating intervention in a previous study [8]. Among postoperative symptoms, loss of appetite, general fatigue, and pain correlated with deterioration in QOL [8]. In previous studies, PC patients with depression reportedly showed associations between significantly aggravated symptoms of fatigue, pain, and anorexia with QOL deterioration [9], and depression in PC patients was considered to be part of the symptomatology with an impact on QOL [10]. There are numerous previous reports on the psychological state of patients for a range of cancers [11-13]. The Reports from studies of PC patients undergoing treatment are significantly important. Though some assessment of the QOL of PC patients has been reported [3, 9, 14-20], no reports show an association between chronological depressive changes and physical symptoms in PC patients postoperatively.

In this study, we aimed to elucidate associations between depressive changes and self-reported, subjective physical symptoms with chronological assessments of depression in PT resection patients pre-operatively, and at 3 and 6 months postoperatively, by a prospective investigation.

\section{Methods}

\section{Participants}

This study is a part of an analysis of an observational study within a prospective cohort study on the correlation between corticotropin-releasing hormone expression and PT prognosis, with a QOL survey. Therefore, the hypothesis of this study and the hypothesis of the main study are completely different. We prospectively recruited patients diagnosed with PT and hospitalized for surgical treatment between January 2103 and December 2015 to obtain assessments of depressive and physical symptoms preoperatively, and postoperatively at months 3 and 6. The analysis set consisted of 101 tumor-resection patients who underwent pancreaticoduodenectomy (PD), distal pancreatectomy (DP), or total pancreatectomy (TP), excluding patients who underwent exploratory bypass laparotomy and non-resected cases.

\section{Materials}

The Self-Rating Depression Scale (SDS) [21, 22] was used to assess depression. The SDS assessment form has 20 items, each of which is rated with a score of 1 to 4 (rarely; sometimes; frequently; always) with higher scores indicating higher levels of depression. According to Zung, the cutoff point for depression is 40. The SDS is used to measure depression in cancer patients [23-26].

Physical symptoms were assessed using the Functional Assessment of Cancer Therapy for Patients with Hepatobiliary Cancer (FACT-Hep) [27, 28], which provides scales for rating responses with regard to treatment and disease progression for hepato-biliary-pancreatic cancers. The FACT-Hep items used were the 18 items from the Hepatobiliary Cancer Subscale (HCS) and three of seven items from the Physical Well-Being (PWB) subscale (hereinafter, "physical symptoms"). Generally, higher HCS and PWB scores indicate better physical condition; however, in this study, we inverted the scores for the relevant HCS (Questions 1, 2, 5, 7, 8, 9, 10, and 12 to 18) and PWB (Questions 19 to 21 ) items to align them with SDS score, which indicates awareness of symptoms. We presented the details in raw form for consistent display of higher scores indicating worse symptoms in this study.

\section{Data analysis}

In this study, the primary endpoint was the chronological depressive score of perioperative PC patients, and secondary endpoints were physical symptoms related to chronological depression.

Chronological changes in SDS scores were compared preoperatively and postoperatively at 3 and 6 months with one-way or two-way ANOVA tests. Patient demographics and physical symptom scores were independent variables and SDS score was a dependent variable. SDS scores of patients with malignant tumors based on histology were further analyzed by categories. When significant interactions were detected, post hoc multiple comparisons were made using the Bonferroni method. Associations between physical symptom scores and SDS score were evaluated with Spearman's rank correlation coefficient. Correlation coefficients $\left(r_{s}\right)$ were interpreted as follows: a magnitude of $r$ between 0.7 and 1.0 indicated "high correlation"; a magnitude of $\mathrm{r}$ between 0.4 and 0.7 indicated "moderate correlation"; a 
magnitude of $\mathrm{r}$ between 0.2 and 0.4 indicated "low correlation", and a magnitude of $\mathrm{r}$ below 0.2 indicated "substantially no correlation". Multiple regression analysis was applied where coefficients were significant in univariate analysis. Symptoms were subjected to entity-relationship modeling preoperatively and postoperatively at 3 and 6 months, with regard to patient symptoms as independent variables and SDS score for the relevant time point as a dependent variable. The distribution of the SDS variable was checked for normality using the Kolmogorov-Smirnov test. All statistical analyses were performed using JMP Pro 12 software (Ver. 12, SAS Institute, Milan, Italy); $p$ values less than 0.05 were considered significant.

\section{Results}

\section{Patient demographics and chronological changes in SDS score}

Subjects were a group of patients being treated for PT. SDS scores were the highest at 3 months after surgery with surgical invasion in many patients.
The analysis set of 101 patients had a mean age of 63.3 \pm 10.7 (28 to 82 ) years [ 53 men $(52.5 \%)$ and 48 women (47.5\%)]. Postoperative histology revealed 73 patients (72.3\%) with malignant tumors and 28 patients (27.7\%) with benign tumors. SDS scores for the 101-patient analysis set were 40.8 preoperatively, 41.9 at postoperative month 3, and 41.5 at postoperative month 6. Patients undergoing PD had a significantly higher SDS score at postoperative month 3 than pre-operation (43.2 vs. 40.9. $p=0.016$ ). Patients with malignant tumors had significantly higher SDS scores at each time point than patients with benign tumors (pre-operation: 41.9 vs. $37.9, p=$ 0.004; postoperative month 3 : 43.5 vs. $37.8, p=0.006$; postoperative month 6 : 42.9 vs. $37.7, p=0.020$ ). SDS scores of patients with malignant tumors were further analyzed by category, with results as follows. Malignant PT patients under 65 years old showed a significantly higher SDS score at postoperative month 3 than postoperative month 6 (44.6 vs. 42.5, $p=0.046$ ). Malignant PT patients undergoing PD showed a significantly higher SDS score at

Table 1 Patient characteristics and SDS scores $n=101$

\begin{tabular}{|c|c|c|c|c|c|}
\hline & & n (\%) & Preop & 3 month postop & 6 month postop \\
\hline \multirow[t]{2}{*}{ Age, years } & under 65 & $47(46.5)$ & 41.32 & 42.87 & 41.94 \\
\hline & 65 or over & $54(53.5)$ & 40.26 & 41.07 & 41.06 \\
\hline \multirow[t]{2}{*}{ Sex } & male & $53(52.5)$ & 40.28 & 42.00 & 42.40 \\
\hline & female & $48(47.5)$ & 41.27 & 41.81 & 40.44 \\
\hline \multirow[t]{3}{*}{ Operation } & PD & $48(47.5)$ & 40.85 & $43.21+$ & 42.23 \\
\hline & DP & $42(41.6)$ & 40.83 & 39.52 & 39.93 \\
\hline & TP & $11(10.9)$ & 40.00 & 45.36 & 44.00 \\
\hline \multirow[t]{6}{*}{ Clavian_Dindo } & 0 & $24(23.8)$ & 38.92 & 39.58 & 40.17 \\
\hline & 1 & $18(17.8)$ & 42.83 & 41.83 & 41.67 \\
\hline & $\|$ & $26(25.7)$ & 40.15 & 41.12 & 39.23 \\
\hline & III a & $28(27.7)$ & 42.71 & 45.25 & 46.07 \\
\hline & III b & $3(3.0)$ & 35.00 & 39.67 & 32.00 \\
\hline & IV a & $2(2.0)$ & 33.00 & 37.50 & 34.00 \\
\hline \multirow[t]{9}{*}{ Histology } & benign & $28(27.7)$ & 37.89 & 37.75 & 37.71 \\
\hline & malignant & $73(72.3)$ & $41.85 * *$ & $43.51 * *$ & $42.92 *$ \\
\hline & under 65 & $32(43.8)$ & 42.56 & 44.59 & $42.47 \neq$ \\
\hline & 65 or over & $41(56.2)$ & 41.29 & 42.66 & 43.24 \\
\hline & male & $42(57.5)$ & 40.83 & 43.19 & 42.93 \\
\hline & female & $31(42.5)$ & 43.23 & 43.94 & 42.87 \\
\hline & PD & $41(56.2)$ & 41.15 & $43.44 \dagger$ & 42.98 \\
\hline & DP & $23(31.5)$ & 43.52 & 42.39 & 41.83 \\
\hline & TP & $9(12.3)$ & 40.78 & 46.67 & 45.33 \\
\hline \multirow[t]{2}{*}{ R status } & RO & $66(90.4)$ & 41.88 & 43.27 & 42.86 \\
\hline & R1 & 7 (9.6) & 41.57 & 45.71 & 43.29 \\
\hline
\end{tabular}

Compared with benign ${ }^{*} p<0.05 * * p<0.01$

Compared with preoperatively $+p<0.05$

Compared with 3 months postoperatively $\neq p<0.05$

$P D$ subtotal stomach-preserving pancreatoduodenectomy, DP distal pancreatectomy, TP total pancreatectomy 
postoperative month 3 than pre-operation (43.4 vs. 41.1, $p=0.028)$ (Table 1$)$.

\section{Chronological changes in subjective physical symptoms} Subjective physical symptoms at postoperative month 3 were worse compared with preoperatively, but many of the symptoms showed improvement at postoperative month 6. Detailed results for subjective physical symptoms are described below.

Hepato-biliary-pancreatic axis physical symptoms: Abilities related to digestion and performing usual activities (scores for "I can digest my food well" and "I am able to do my usual activities", respectively) were significantly improved at postoperative month 3 versus preoperatively ( $p<$ $0.05)$. Many items showed significant deterioration in physical symptoms at postoperative month 3 versus preoperatively; these were the scores for "I have swelling or cramps in my stomach area" $(p<0.001)$, "I feel fatigued" $(p<0.05)$, and "I have had a change in the way food tastes" $(p<0.05)$. Each of these three items then showed significant improvement at postoperative month 6 versus postoperative month 3 ( $p<0.001, p<0.001$, and $p<0.01$, respectively). Scores for the following items were significantly improved at postoperative month 6 versus postoperative month 3 (after they had tended to show deterioration in physical symptoms at postoperative month 3): "I am losing weight" ( $p<0.01)$, "I have diarrhea" $(p<0.05)$, "I am unhappy about a change in my appearance" $(p<0.05)$, "I am bothered by constipation" $(p<0.001)$, "I am bothered by jaundice or yellow color to my skin" ( $p<0.001)$, "I have had fevers" $(p<0.001)$, "I have had chills" ( $p<0.01)$, and "I have discomfort or pain in my stomach area" $(p<0.01)$ (Fig. 1)

General physical symptoms: Scores for "I have a lack of energy" $(p<0.01)$ and "I have pain" $(p<0.01)$ ] showed significant deterioration at postoperative month 3 . Both these items showed significant improvement at postoperative month 6 versus postoperative month 3 ( $p<0.001$ for both items) (Fig. 1)

\section{Associations between physical symptoms and SDS score} Many time-dependent associations were found between physical symptoms and SDS score.

In detail, at pre-operation, the scores for the following six items showed significant correlations with SDS score "I have control of my bowels", "I can digest my food well", "I am able to do my usual activities", "I have a lack of energy", "I have nausea", and "I have pain"; these correlations were graded as "low" to "moderate" $(p<0.000-p<0.05)$. At postoperative month 6 , the scores for the following six items showed significant correlation with SDS score: "I can digest my food well", "I have a good appetite", "I am able to do my usual activities", "I have a lack of energy", "I have nausea", and "I have pain"; these correlations were graded as "low" to "moderate" $(p<0.000-p<0.05)$. At postoperative month 6 , the following 16 items showed a significant correlation with SDS score: "I have swelling or cramps in my stomach area", "I am losing weight", "I have control of my bowels", "I can digest my food well", "I have a good appetite", "I am unhappy about a change in my appearance", "I feel fatigued", "I am able to do my usual activities", "I have had fevers", "I have had itching", "I have had a change in the way food tastes", "I have had chills", "My mouth is dry,", "I have discomfort or pain in my stomach area", "I have a lack of energy", and "I have pain"; these correlations were graded as "low" to "moderate" ( $p<$ $0.000-p<0.05$ ) (Table 2)

Physical symptoms that were significantly correlated with SDS score were subjected to multiple regression analysis, and the results are stated below.

Preoperatively, significant associations were noted for the following two items: "I have a lack of energy" ( $p<$ $0.000)$ and "I have pain" ( $p=0.018)\left[\mathrm{R}^{2}=0.43\right.$; Table 3].

At postoperative month 3 , significant associations were noted for the following two items: "I am able to do my usual activities" ( $p=0.031)$ and "I have a lack of energy" $(p<0.000)\left[\mathrm{R}^{2}=0.51\right.$; Table 4$]$.

At postoperative month 6, significant associations were noted for the following two items: "I have swelling or cramps in my stomach area" $(p=0.034)$ and "I have control of my bowels" ( $(p=0.049)\left[\mathrm{R}^{2}=0.52\right.$; Table 5].

\section{Discussion}

\section{Characteristics of depression in PT resection patients}

The PT patients in this study were generally considered to be persistently depressed preoperatively through postoperative month 6 . In particular, patients who received a postoperative histological diagnosis of malignancy were highly depressed; this finding possibly reflected concerns over the diagnosis, in the same way as has been reported for patients with other cancers $[10,29,30]$. PC has a poor prognosis, and survival rates are poor even when operable [1]. Intraductal papillary mucinous neoplasm is a risk factor for PC even though such tumors are benign [31-33]. Many patients in this study were warned of the likelihood of a malignant tumor prior to undergoing surgery, and the patients who received such a warning might have felt psychological distress concerning the operation and their future and developed tendencies to depression. Even patients who can resume normal living postoperatively could have had fears of relapse and metastasis. In addition to those fears, they may feel a sense of loss and loneliness in their daily lives, and effects could have been attributable to the resultant psychological experience [10, 34]. Levels of depression were high among working-age (under 65 years old) patients with malignant tumors at postoperative month 3 , and anxiety about the future has been suggested as likely in this group of cancer patients [35]. 


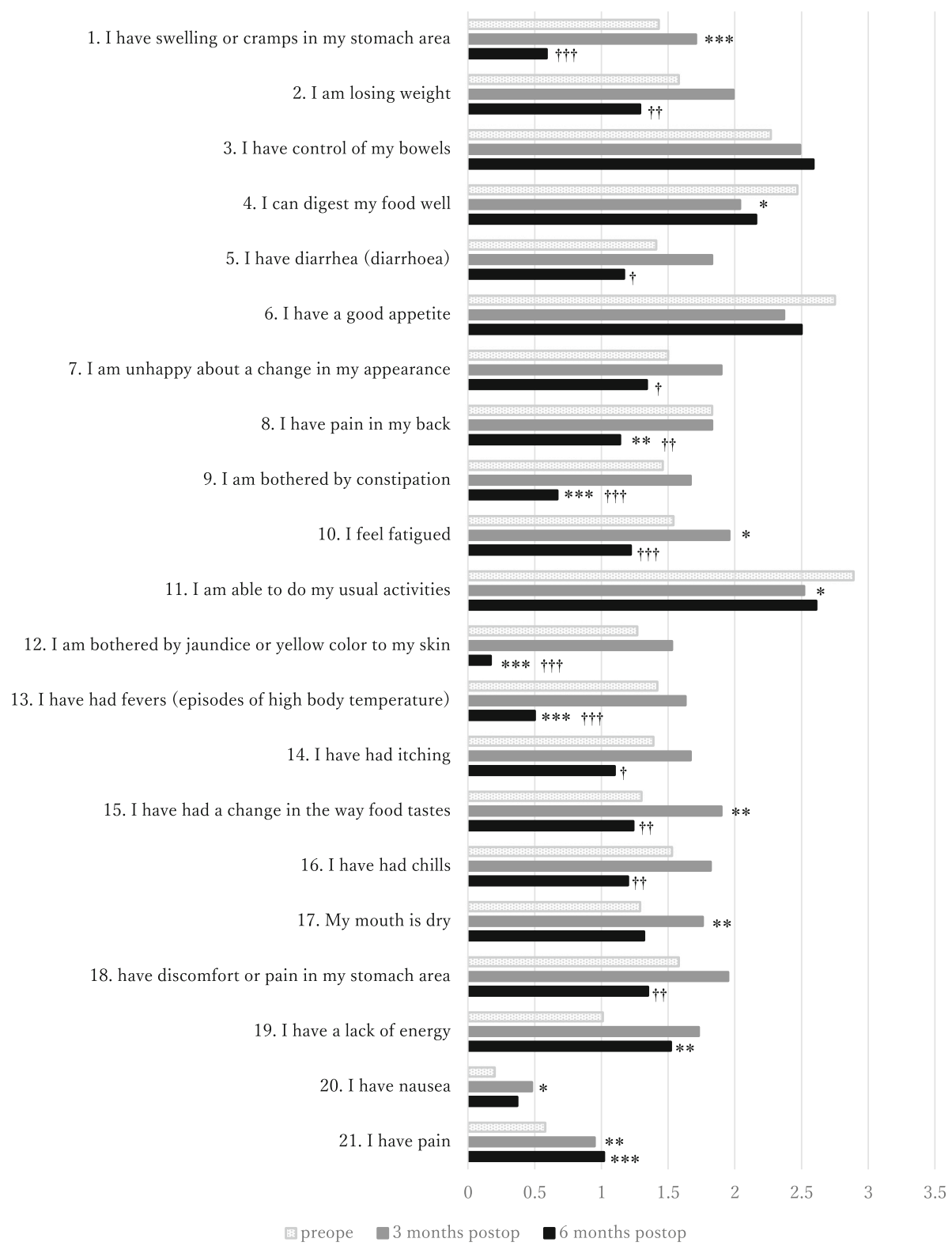

Fig. 1 Chronological changes in subjective physical symptoms scores preoperation, post 3 and 6 months operation. Scores were reversed for items $1,2,5,7,8,9,10,12,13,14,15,16,17,18,19,20$, and 21. High symptom score was unified to show poor condition. Compared with preoperation ${ }^{*} p<0.05,{ }^{* *} p<0.01,{ }^{* * *} p<0.001$. Compared with post 3 month operation $+p<0.05,+\dagger p<0.01,++\uparrow p<0.001$. Subjective physical symptoms at postoperative month 3 were worse compared with preoperatively, but many of the symptoms showed improvement at postoperative month 6

\section{Chronological changes in associations between depression and physical symptoms}

The symptoms related to the depression of patients with pancreatic tumor were chronologically distinctive, which may reflect the physical and mental conditions experienced time dependently from surgery. PT patients scheduled for surgery showed depression preoperatively, and this depression was influenced by lack of energy and pain (physical symptom scores for "I have a lack of energy" and "I have pain", respectively.) Inability to perform usual activities and lack of energy (scores for "I am able to do my usual activities" and "I have a lack of energy", respectively) were influencing factors for postoperative depression during the period when surgery-related stress was immense. Gastrointestinal symptoms were the main physical symptoms with delayed manifestation, and were suggested to be possible subsequent causative factors for depression. Postoperative depression was high in patients who underwent PD and at postoperative month 3 ; items related to gastrointestinal symptoms and digestion, and those reflecting surgical stress 
Table 2 Correlation between symptoms and SDS score

\begin{tabular}{|c|c|c|c|}
\hline & $\operatorname{SDS}\left(r_{s}\right)$ & $3 \mathrm{M} \mathrm{SDS}\left(r_{\mathrm{s}}\right)$ & $6 \mathrm{M} \mathrm{SDS}\left(r_{\mathrm{s}}\right)$ \\
\hline 1. I have swelling or cramps in my stomach area & -.016 & -.070 & $.384^{* * *}$ \\
\hline 2. I am losing weight & -.065 & -.117 & $.370^{* * *}$ \\
\hline 3. I have control of my bowels & $-.291^{* *}$ & -.165 & $-.329^{* * *}$ \\
\hline 4. I can digest my food well & $-.255^{*}$ & $-.330^{* * *}$ & $-.436^{* * *}$ \\
\hline 5. I have diarrhea (diarrhoea) & -.070 & -.043 & .130 \\
\hline 6. I have a good appetite & -.170 & $-.378^{* * *}$ & $-.354^{* * *}$ \\
\hline 7. I am unhappy about a change in my appearance & -.031 & .038 & $.274^{* *}$ \\
\hline 8. I have pain in my back & .041 & -.143 & .073 \\
\hline 9. I am bothered by constipation & .011 & -.034 & .060 \\
\hline 10. I feel fatigued & -.063 & .002 & $.538^{* * *}$ \\
\hline 11. I am able to do my usual activities & $-.295^{* *}$ & $-.400^{* * *}$ & $-.330^{* * *}$ \\
\hline 12. I am bothered by jaundice or yellow color to my skin & -.044 & -.099 & .104 \\
\hline 13. I have had fevers (episodes of high body temperature) & -.011 & -.034 & $.205^{*}$ \\
\hline 14. I have had itching & -.005 & -.031 & $.220^{*}$ \\
\hline 15. I have had a change in the way food tastes & -.031 & .039 & $.292^{* *}$ \\
\hline 16. I have had chills & .025 & -.102 & $.287^{* *}$ \\
\hline 17. My mouth is dry & -.056 & -.084 & $.284^{* *}$ \\
\hline 18. have discomfort or pain in my stomach area & .006 & .031 & $.392^{* * *}$ \\
\hline 19. I have a lack of energy & $.567^{* * *}$ & $.659^{* * *}$ & $.498^{* * *}$ \\
\hline 20. I have nausea & $.209^{*}$ & $.277^{* *}$ & .159 \\
\hline 21. I have pain & $.449^{* * *}$ & $.370^{* * *}$ & $.348^{* * *}$ \\
\hline
\end{tabular}

Spearman's rank correlation coefficient $\left(\mathrm{r}_{\mathrm{s}}\right){ }^{*} p<0.05{ }^{* *} p<0.01{ }^{* * *} p<0.001$

showed increased scores at postoperative month 3. Accordingly, it was suggested that the extent of surgery-related stress and postoperative physical symptoms possibly affected psychological state. PD involves extensive surgical techniques, prolonged duration of surgery, and a tendency for massive hemorrhage. Likely postoperative complications include intractable diarrhea, pancreatic exocrine insufficiency, and gastrointestinal dysfunction, depending on the extent of the resection [36-39]. Patients with malignant tumors may receive adjuvant chemotherapy when necessary [40, 41], which imposes additional stress to that from surgery. As a result, patients frequently experience toxic effects and manifest gastrointestinal symptoms such as diarrhea and anorexia $[41,42]$. These physical symptoms were also suggested to have possibly influenced patients' psychological state in this study. Most subjective physical symptoms in this area showed dramatic improvement at postoperative month 6. However, we considered that this improvement was due to gradual physical and mental adaption to daily life by the patients, control of blood glucose, and dietary intake amount and content suitable for the patient in question, as well as being an improvement in gastrointestinal symptoms. However, we identified one point that requires attention. The greatest number of associations between subjective

Table 3 Model of relationship between symptoms and depression preoperatively

\begin{tabular}{|c|c|c|c|c|}
\hline & $\mathrm{B}$ & $\beta$ & $P$ value & $95 \% \mathrm{Cl}[\mathrm{LL}, \mathrm{UL}]$ \\
\hline & 41.12 & & $<.000$ & {$[36.34,45.90]$} \\
\hline 3. I have control of my bowels & -0.90 & -0.14 & 0.110 & {$[-2.01,0.21]$} \\
\hline 4. I can digest my food well & 0.10 & 0.01 & 0.876 & {$[1.37,-1.17]$} \\
\hline 11. I am able to do my usual activities & -1.11 & -0.15 & 0.076 & {$[0.12,-2.33]$} \\
\hline 19. I have a lack of energy & 3.26 & 0.42 & $<.000$ & {$[4.58,1.94]$} \\
\hline 20. I have nausea & 1.31 & 0.02 & 0.859 & {$[2.83,-2.36]$} \\
\hline \multirow[t]{2}{*}{ 21. I have pain } & 2.20 & 0.22 & 0.018 & {$[4.01,0.39]$} \\
\hline & & & & $=0.43, p<.0001$ \\
\hline
\end{tabular}


Table 4 Model of relationship between symptoms and depression at 3 months postoperatively

\begin{tabular}{|c|c|c|c|c|}
\hline & B & $\beta$ & $P$ value & $95 \% \mathrm{Cl}[\mathrm{LL}, \mathrm{UL}]$ \\
\hline & 37.78 & & $<.000$ & {$[31.69,43.88]$} \\
\hline 4. I can digest my food well & -0.76 & -0.10 & 0.269 & {$[-2.16,0.60]$} \\
\hline 6. I have a good appetite & -0.02 & -0.002 & 0.982 & {$[1.48,1.44]$} \\
\hline 11. I am able to do my usual activities & -1.44 & -0.17 & 0.031 & {$[-2.75,-0.14]$} \\
\hline 19. I have a lack of energy & 4.32 & 0.52 & $<.000$ & {$[2.97,5.67]$} \\
\hline 20. I have nausea & 0.85 & 0.07 & 0.387 & {$[-1.09,2.78]$} \\
\hline \multirow[t]{2}{*}{ 21. I have pain } & 1.55 & 0.14 & 0.076 & {$[-0.17,3.26]$} \\
\hline & & & & $=0.51, p<.0001$ \\
\hline
\end{tabular}

Cl confidence interval, LL lower limit, UL upper limit

physical symptoms and depression was noted at postoperative month 6 . We suggest that approaches to monitor and counteract depression are particularly necessary for patients with persistent symptoms 6 months postoperatively and those with severe symptoms.

\section{Implications}

This study demonstrates that surgical invasiveness possibly affects depression in patients with PC. PT patients are reported to often experience fatigue, lower back pain, and digestive system dysfunction [43]. We also identified these physical symptoms in this study and elucidated them as factors that influence depression. Depression in cancer patients generally leads to impairment of QOL, and is linked to more than just physical symptoms, anxiety, and pain; it is associated with reduced adherence to cancer therapy and prolonged hospital stays [10], with the imposition of mental burden on caregivers [44]. Measures for preventing and dealing with depression in cancer patients are extremely important. Depression is reportedly greater in PC patients $[9,45-$ 47] than those with advanced gastrointestinal carcinomas such as stomach and colorectal cancer [48]. Approaches can cover a number of specific points. A symptom management approach that emphasizes gastrointestinal symptoms should be initiated prior to surgery. Care and drug therapies for alleviating these physical symptoms should be provided in the long term as required. Patients should be encouraged to engage in more physical activity [49]. Depression is closely related to QOL and it has been reported that depression interventions including psychoeducational interventions improve QOL $[19,50]$. Care that involves supportive-expressive discussion [51], counseling, and psychological support

Table 5 Model of relationship between symptoms and depression at 6 months postoperatively

\begin{tabular}{|c|c|c|c|c|}
\hline & $B$ & $\beta$ & $P$ value & $95 \% \mathrm{Cl}[\mathrm{LL}, \mathrm{UL}]$ \\
\hline & 41.41 & & $<.000$ & {$[35.54,47.27]$} \\
\hline 1. I have swelling or cramps in my stomach area & 2.52 & 0.23 & 0.034 & {$[0.19,4.86]$} \\
\hline 2. I am losing weight & 1.22 & 0.18 & 0.050 & {$[-0.00,2.44]$} \\
\hline 3. I have control of my bowels & -1.46 & -0.19 & 0.049 & {$[-2.91,-0.01]$} \\
\hline 4. I can digest my food well & -0.88 & -0.11 & 0.293 & {$[-2.52,0.77]$} \\
\hline 6. I have a good appetite & -0.82 & -0.11 & 0.263 & {$[-2.25,0.62]$} \\
\hline 7. I am unhappy about a change in my appearance & -0.15 & -0.02 & 0.845 & {$[-1.63,1.34]$} \\
\hline 10. I feel fatigued & 1.80 & 0.20 & 0.089 & {$[-0.28,3.87]$} \\
\hline 11. I am able to do my usual activities & -0.10 & -0.01 & 0.890 & {$[-1.60,1.39]$} \\
\hline 13. I have had fevers (episodes of high body temperature) & -0.84 & -0.08 & 0.425 & {$[-2.93,1.25]$} \\
\hline 14. I have had itching & 0.81 & 0.010 & 0.266 & {$[-0.63,2.24]$} \\
\hline 15. I have had a change in the way food tastes & -0.51 & -0.08 & 0.467 & {$[-1.91,0.88]$} \\
\hline 16. I have had chills & -0.02 & -0.00 & 0.980 & {$[-1.46,1.43]$} \\
\hline 17. My mouth is dry & 0.26 & 0.04 & 0.730 & {$[-1.24,1.76]$} \\
\hline 18. I have discomfort or pain in my stomach area & 0.42 & 0.05 & 0.641 & {$[-1.38,2.23]$} \\
\hline 19. I have a lack of energy & 1.34 & 0.17 & 0.151 & {$[-0.50,3.19]$} \\
\hline \multirow[t]{2}{*}{ 21. I have pain } & 0.20 & 0.02 & 0.844 & {$[-1.82,2.22]$} \\
\hline & & & & $\mathrm{R}^{2}=0.52, p<.0001$ \\
\hline
\end{tabular}


$[50,52]$ should be provided. Attention to these measures may promote alleviate psychological symptoms and improved physical condition [19]. Based on these reports and the findings in this study, we suggest that proactive symptom management and psychological support is vital for PT patients, especially those with malignant tumors.

\section{Limitations}

First, pancreatic resection involves a high degree of surgery-related stress. In this study, we elucidated the influence of subjective physical symptoms of that stress on depression. Among the patients we evaluated, patients with histologically diagnosed malignant tumors were significantly more depressed than patients with benign tumors. We consider that prognosis-related anxiety may be particularly reflected in depression when the tumor is malignant. PC has a poor prognosis, and the psychological burden should be fully considered in any evaluation. Second, we could not evaluate the influence of diabetes mellitus and insulin supplementation on depression. Future studies with long-term follow up should thus be carried out and to assess long-term effects including QOL. Third, for many patients PC is detected too late for surgery to be effective, and survival is limited even for patients who undergo surgery. There are limits on the parameters that can be investigated in a single center, and a detailed investigation was not possible in this study. Further investigations using multi-center studies are needed for future research on depression in PC patients.

\section{Conclusions}

PC patients experience persistently high levels of depression preoperatively through 6 months postoperatively. Psychological support is needed both pre- and postoperatively, especially for patients with malignant tumors, those undergoing PD, and those under 65 years of age. Several physical symptoms are aggravated due to pancreatic resection between the preoperative stage and 3 months postoperatively, and then are improved at 6 months postoperatively. Pain and gastrointestinal symptoms are the major subjective physical symptoms possibly associated with depression. We consider that depression in PC patients could be reduced by ensuring long-term symptom management commencing prior to surgery and by psychiatric interventions tailored to patient characteristics.

\section{Abbreviations}

DP: Distal pancreatectomy; PD: Subtotal stomach-preserving pancreatoduodenectomy; TP: Total pancreatectomy

\section{Acknowledgements}

The authors appreciate the data collection and suggestions provided by Tohoku University Hospital Hepato-Biliary Pancreatic Surgical team, Ms. Masami Sato, Ms. Hiroko Watanabe, and Ms. Emiko Shibuya (Department of Surgery, Tohoku University Graduate School of Medicine, Sendai, Japan). Without their selfless contribution and hard work, this study would not have been possible.
Funding

This work was supported by JSPS KAKENHI Grant Number JP16K07140.

Availability of data and materials

Due to the conditions outlined in the ethics approval and identifiable information included in the data file and survey materials, these items have not been made available for review.

\section{Authors' contributions}

All authors contributed equally to the project design, data collection, data analysis, and manuscript preparation. All authors read and approved the final manuscript.

\section{Ethics approval and consent to participate}

This study was approved by the Ethics Committee of Tohoku University Graduate School of Medicine (IRB No.2012-1-403). A participant information statement and consent form were provided and verbally explained to each participant. Participation was voluntary, and participants were allowed time to read the forms prior to the commencement of the survey. Participation in the survey was anonymous and did not affect an employees' relationship with the employer.

Consent for publication

Not applicable.

\section{Competing interests}

The authors declare that they have no competing interests.

\section{Publisher's Note}

Springer Nature remains neutral with regard to jurisdictional claims in published maps and institutional affiliations.

\section{Author details}

${ }^{1}$ Department of Oncology Nursing, Tohoku University Graduate School of Medicine, Sendai, Japan. '2Department of Nursing, Tohoku University School of Health Sciences, Sendai, Japan. ${ }^{3}$ Department of Surgery, Tohoku University Graduate School of Medicine, Sendai, Japan. ${ }^{4}$ Department of Behavioral Medicine, Tohoku University Graduate School of Medicine, Sendai, Japan. ${ }^{5}$ Department of Oncology Nursing, Tohoku University, 2-1 Seiryo-machi, Aoba-ku, Sendai 980-8575, Japan.

Received: 20 March 2018 Accepted: 11 September 2018

Published online: 28 September 2018

References

1. Siegel RL, Miller KD, Jemal A. Cancer statistics, 2018. CA Cancer J Clin 2018. doi: https://doi.org/10.3322/caac.21442. PubMed PMID: 29313949.

2. Doi R, Imamura M, Hosotani R, Imaizumi T, Hatori T, Takasaki K, et al. Surgery versus radiochemotherapy for resectable locally invasive pancreatic cancer: final results of a randomized multi-institutional trial. Surg Today 2008;38(11):10211028. doi: https://doi.org/10.1007/s00595-007-3745-8. PubMed PMID: 18958561

3. Heerkens HD, Tseng DS, Lips IM, van Santvoort HC, Vriens MR, Hagendoorn J, et al. Health-related quality of life after pancreatic resection for malignancy. Br J Surg 2016;103(3):257-266. Epub 2016/01/ 21. doi: https://doi.org/10.1002/bjs.10032. PubMed PMID: 26785646.

4. McKay A, Sutherland FR, Bathe OF, Dixon E. Morbidity and mortality following multivisceral resections in complex hepatic and pancreatic surgery. J Gastrointest Surg 2008;12(1):86-90. doi: https://doi.org/10.1007/ s11605-007-0273-1. PubMed PMID: 17710505.

5. Gumbs AA, Gres P, Madureira FA, Gayet B. Laparoscopic vs. open resection of noninvasive intraductal pancreatic mucinous neoplasms. J Gastrointest Surg 2008;12(4):707-712. doi: https://doi.org/10.1007/s11605-007-0311-z. PubMed PMID: 17909923.

6. Pratt W, Joseph S, Callery MP, Vollmer CM, Jr. POSSUM accurately predicts morbidity for pancreatic resection. Surgery 2008;143(1):8-19. doi: https://doi. org/10.1016/j.surg.2007.07.035. PubMed PMID: 18154928.

7. Wang $H$, Chen $T$, Wang $H$, Song Y, Li X, Wang J. A systematic review of the physiological and operative severity score for the enUmeration of mortality and morbidity and its Portsmouth modification as predictors of post-operative morbidity and mortality in patients undergoing pancreatic surgery. Am J Surg 2013;205(4):466-472. doi: https://doi.org/10.1016/j.amjsurg.2012.06.011. PubMed PMID: 23395580 
8. Sato N, Katayose Y, Motoi F, Nakagawa K, Sakata N, Kawaguchi K, et al. Strategy of Symptom-Targeted Intervention Based on Patient Quality of Life at Three Months After Pancreatectomy. Suizo. 2015;30(5):654-62. doi: https://doi.org/10.2958/suizo.30.654

9. Jia L, Jiang SM, Shang YY, Huang YX, Li YJ, Xie DR, et al. Investigation of the incidence of pancreatic cancer-related depression and its relationship with the quality of life of patients. Digestion 2010;82(1):4-9. Epub 2010/02/11. doi: https://doi.org/10.1159/000253864. PubMed PMID: 20145402.

10. Tang CC, Von Ah D, Fulton JS. The symptom experience of patients with advanced pancreatic Cancer: an integrative review. Cancer Nurs 2018;41(1): 33-44. Epub 2017/01/07. doi: https://doi.org/10.1097/ncc. 0000000000000463. PubMed PMID: 28059839.

11. Kim GM, Kim SJ, Song SK, Kim HR, Kang BD, Noh SH, et al. Prevalence and prognostic implications of psychological distress in patients with gastric cancer. BMC cancer. 2017;17(1):283. Epub 2017/04/22. doi: https://doi.org/10. 1186/s12885-017-3260-2. PubMed PMID: 28427439; PubMed Central PMCID: PMCPMC5399416.

12. Vehling S, Mehnert A, Hartmann M, Oing C, Bokemeyer C, Oechsle K. Anxiety and depression in long-term testicular germ cell tumor survivors. Gen Hosp Psychiatry 2016;38:21-25. Epub 2015/10/07. doi: https://doi.org/ 10.1016/j.genhosppsych.2015.09.001. PubMed PMID: 26439320.

13. Scheffold K, Philipp R, Koranyi S, Engelmann D, Schulz-Kindermann F, Harter $M$, et al. Insecure attachment predicts depression and death anxiety in advanced cancer patients. Palliat Support Care 2017:1-9. Epub 2017/05/16. doi: https://doi.org/10.1017/s1478951517000281. PubMed PMID: 28502270

14. Bauer MR, Bright EE, MacDonald JJ, Cleary EH, Hines OJ, Stanton AL. Quality of Life in Patients With Pancreatic Cancer and Their Caregivers: A Systematic Review. Pancreas. 2018;47(4):368-375. doi: https://doi.org/10.1097/Mpa. 0000000000001025. PubMed PMID: WOS:000428083700009.

15. Braun DP, Gupta D, Staren ED. Longitudinal health-related quality of life assessment implications for prognosis in stage IV pancreatic Cancer. Pancreas. 2012;00(00):1-6.

16. Velanovich $\mathrm{V}$. The association of quality-of-life measures with malignancy and survival in patients with pancreatic pathology. Pancreas 2011;40(7): 1063-1069. Epub 2011/07/26. doi: https://doi.org/10.1097/MPA. Ob013e31821ad8eb. PubMed PMID: 21785386.

17. Velanovich V, Wollner I. Quality of life and performance status in patients with pancreatic and periampullary tumors. Int J Clin Oncol 2011;16(4):401407. Epub 2011/02/25. doi: https://doi.org/10.1007/s10147-011-0200-z. PubMed PMID: 21347628.

18. Eaton AA, Gonen M, Karanicolas P, Jarnagin WR, D'Angelica MI, DeMatteo R, et al. Health-Related Quality of Life After Pancreatectomy: Results From a Randomized Controlled Trial. Annals of surgical oncology. 2016;23(7):213745. Epub 2016/01/21. doi: https://doi.org/10.1245/s10434-015-5077-z. PubMed PMID: 26786091; PubMed Central PMCID: PMCPMC4891251.

19. Sugimoto H, Kawashima H, Ohno E, Hayashi D, Kuwahara T, Morishima T, et al. The prognostic factors and trajectory of HRQOL in patients with pancreatic cancer who received psychiatric intervention. J Gastroenterol Hepatol 2016;31(3):685-690. Epub 2015/09/29. doi: https://doi.org/10.1111/ jgh.13172. PubMed PMID: 26412310

20. Jimenez-Fonseca P, Carmona-Bayonas A, Martin-Perez E, Crespo G, Serrano $R$, Llanos $M$, et al. Health-related quality of life in well-differentiated metastatic gastroenteropancreatic neuroendocrine tumors. Cancer Metastasis Rev 2015;34(3):381-400. Epub 2015/08/08. doi: https://doi.org/10. 1007/s10555-015-9573-1. PubMed PMID: 26245646.

21. Zung WW. A self-rating depression scale. Arch Gen Psychiatry 1965;12:6370. PubMed PMID: 14221692

22. Fukuda K, Kobayashi S. A study on a self-rating depression scale. Seishin Shinkeigaku Zasshi. 1973;75:673-9.

23. Zhang J, Zhou Y, Feng Z, Xu Y, Zeng G. Longitudinal trends in anxiety, depression, and quality of life during different intermittent periods of adjuvant breast Cancer chemotherapy. Cancer Nurs 2018;41(1):62-68. Epub 2016/12/07. doi: https://doi.org/10.1097/ncc.0000000000000451. PubMed PMID: 27922916

24. Sharpley CF, Christie DR, Bitsika V, Miller BJ. Trajectories of total depression and depressive symptoms in prostate cancer patients receiving six months of hormone therapy. Psycho-Oncology 2017;26(1):60-66. Epub 2016/02/10. doi: https://doi.org/10.1002/pon.4100. PubMed PMID: 26857160.

25. Guo $X, X u$ J, Ying $E$, Yu Z, Sun T. Correlation between hormone receptor status and depressive symptoms in patients with metastatic breast cancer.
Oncotarget. 2017;8(31):50774-50781. Epub 2017/09/09. doi: https://doi.org/ 10.18632/oncotarget.15037. PubMed PMID: 28881602; PubMed Central PMCID: PMCPMC5584203.

26. Chen J, Li W, Cui L, Qian Y, Zhu Y, Gu H, et al. Chemotherapeutic Response and Prognosis among Lung Cancer Patients with and without Depression. J Cancer. 2015;6(11):1121-1129. Epub 2015/10/31. doi: https://doi.org/10. 7150/jca.11239. PubMed PMID: 26516360; PubMed Central PMCID: PMCPMC4615348.

27. Heffernan N, Cella D, Webster K, Odom L, Martone M, Passik S, et al. Measuring health-related quality of life in patients with hepatobiliary cancers: the functional assessment of cancer therapy-hepatobiliary questionnaire. J Clin Oncol 2002;20(9):2229-2239. Epub 2002/05/01. PubMed PMID: 11980994.

28. Yount S, Cella D, Webster K, Heffernan N, Chang C, Odom L, et al. Assessment of patient-reported clinical outcome in pancreatic and other hepatobiliary cancers: the FACT hepatobiliary symptom index. J Pain Symptom Manag 2002;24(1):32-44. Epub 2002/08/17. PubMed PMID: 12183093.

29. Diaz-Frutos D, Baca-Garcia E, Garcia-Foncillas J, Lopez-Castroman J. Predictors of psychological distress in advanced cancer patients under palliative treatments. Eur J Cancer Care (Engl) 2016;25(4):608-615. Epub 2016/06/09. doi: https://doi.org/10.1111/ecc.12521. PubMed PMID: 27271213.

30. Zhu L, Ranchor AV, Helgeson VS, van der Lee M, Garssen B, Stewart RE, et al. Benefit finding trajectories in cancer patients receiving psychological care: predictors and relations to depressive and anxiety symptoms. Br J Health Psychol 2018;23(2):238-252. Epub 2017/11/16. doi: https://doi.org/10.1111/ bjhp.12283. PubMed PMID: 29139593.

31. Yamaguchi K, Kanemitsu S, Hatori T, Maguchi H, Shimizu Y, Tada M, et al. Pancreatic ductal adenocarcinoma derived from IPMN and pancreatic ductal adenocarcinoma concomitant with IPMN. Pancreas 2011:40(4):571-580. doi: https://doi.org/10.1097/MPA.0b013e318215010c. PubMed PMID: 21499212.

32. Maguchi H, Tanno S, Mizuno N, Hanada K, Kobayashi G, Hatori T, et al. Natural history of branch duct intraductal papillary mucinous neoplasms of the pancreas: a multicenter study in Japan. Pancreas 2011;40(3):364-370. doi: https://doi.org/10.1097/MPA.0b013e31820a5975. PubMed PMID: 21289527.

33. Kobayashi G, Fujita N, Maguchi H, Tanno S, Mizuno N, Hanada K, et al. Natural history of branch duct intraductal papillary mucinous neoplasm with mural nodules: a Japan Pancreas Society multicenter study. Pancreas. 2014;43(4):532-8. doi: https://doi.org/10.1097/MPA.0000000000000080. PubMed PMID: 24717801; PubMed Central PMCID: PMCPMC4206346.

34. Deckx L, van den Akker M, van Driel M, Bulens P, van Abbema D, TjanHeijnen $V$, et al. Loneliness in patients with cancer: the first year after cancer diagnosis. Psycho-Oncology 2015;24(11):1521-1528. Epub 2015/04/29. doi: https://doi.org/10.1002/pon.3818. PubMed PMID: 25914244.

35. Muramatsu K, Matsuda S, Hayashida K, Kubo T, Fujino Y, Fujimori K, et al. Do the Japanese Cancer patients receive an appropriate psychiatric support at the acute care hospitals? —an evaluation trial by DPC based data-. Asian Pacific J Dis Manage. 2011;5(1):13-7.

36. Diener MK, Fitzmaurice C, Schwarzer G, Seiler CM, Antes G, Knaebel HP, et al. Pylorus-preserving pancreaticoduodenectomy (pp Whipple) versus pancreaticoduodenectomy (classic Whipple) for surgical treatment of periampullary and pancreatic carcinoma. Cochrane Database Syst Rev 2011; (5):CD006053. doi: https://doi.org/10.1002/14651858.CD006053.pub4. PubMed PMID: 21563148.

37. Karanicolas PJ, Davies E, Kunz R, Briel M, Koka HP, Payne DM, et al. The pylorus: take it or leave it? Systematic review and meta-analysis of pyloruspreserving versus standard whipple pancreaticoduodenectomy for pancreatic or periampullary cancer. Ann Surg Oncol 2007;14(6):1825-1834. doi: https://doi.org/10.1245/s10434-006-9330-3. PubMed PMID: 17342566.

38. Kawai M, Tani M, Hirono S, Miyazawa M, Shimizu A, Uchiyama K, et al. Pylorus ring resection reduces delayed gastric emptying in patients undergoing pancreatoduodenectomy: a prospective, randomized, controlled trial of pylorus-resecting versus pylorus-preserving pancreatoduodenectomy. Ann Surg 2011;253(3):495-501. doi: https://doi. org/10.1097/SLA.0b013e31820d98f1. PubMed PMID: 21248633.

39. Matsumoto I, Shinzeki M, Asari S, Goto T, Shirakawa S, Ajiki T, et al. A prospective randomized comparison between pylorus- and subtotal stomach-preserving pancreatoduodenectomy on postoperative delayed gastric emptying occurrence and long-term nutritional status. I Surg Oncol 2014:109(7):690-696. doi: https://doi.org/10.1002/jso.23566. PubMed PMID: 24619624 
40. Saung MT, Zheng L. Current Standards of Chemotherapy for Pancreatic Cancer. Clinical therapeutics. 2017;39(11):2125-34. Epub 2017/09/25. doi: https://doi.org/10.1016/j.clinthera.2017.08.015. PubMed PMID: 28939405; PubMed Central PMCID: PMCPMC5705388.

41. Uesaka K, Boku N, Fukutomi A, Okamura Y, Konishi M, Matsumoto I, et al. Adjuvant chemotherapy of S-1 versus gemcitabine for resected pancreatic cancer: a phase 3, open-label, randomised, non-inferiority trial (JASPAC 01) Lancet 2016;388(10041):248-257. doi: https://doi.org/10.1016/S01406736(16)30583-9. PubMed PMID: 27265347.

42. Van Laethem JL, Hammel P, Mornex F, Azria D, Van Tienhoven G, Vergauwe $P$, et al. Adjuvant gemcitabine alone versus gemcitabine-based chemoradiotherapy after curative resection for pancreatic cancer: a randomized EORTC-40013-22012/FFCD-9203/GERCOR phase II study. J Clin Oncol. 2010;28(29):4450-4456. Epub 2010/09/15. doi: https://doi.org/10. 1200/jco.2010.30.3446. PubMed PMID: 20837948; PubMed Central PMCID: PMCPMC2988636.

43. Cloyd JM, Tran Cao HS, Petzel MQ, Denbo JW, Parker NH, Nogueras-Gonzalez GM, et al. Impact of pancreatectomy on long-term patient-reported symptoms and quality of life in recurrence-free survivors of pancreatic and periampullary neoplasms. J Surg Oncol 2017;115(2):144-150. Epub 2016/11/20. doi: https:// doi.org/10.1002/jso.24499. PubMed PMID: 27859270.

44. Janda M, Neale RE, Klein K, O'Connell DL, Gooden H, Goldstein D, et al. Anxiety, depression and quality of life in people with pancreatic cancer and their carers. Pancreatology 2017. Epub 2017/02/06. doi: https://doi.org/10. 1016/j.pan.2017.01.008. PubMed PMID: 28153446.

45. Brintzenhofe-Szoc KM, Levin TT, Li Y, Kissane DW, Zabora JR. Mixed anxiety/ depression symptoms in a large cancer cohort: prevalence by cancer type. Psychosomatics 2009;50(4):383-391. Epub 2009/08/19. doi: https://doi.org/ 10.1176/appi.psy.50.4.383. PubMed PMID: 19687179.

46. Clark KL, Loscalzo M, Trask PC, Zabora J, Philip EJ. Psychological distress in patients with pancreatic cancer--an understudied group. Psycho-Oncology 2010;19(12):1313-1320. Epub 2010/02/02. doi: https://doi.org/10.1002/pon. 1697. PubMed PMID: 20119937.

47. Carney CP, Jones L, Woolson RF, Noyes R, Jr., Doebbeling BN. Relationship between depression and pancreatic cancer in the general population. Psychosom Med 2003;65(5):884-888. PubMed PMID: 14508036.

48. Matsushita T, Matsushima E, Maruyama M. Anxiety and depression of patients with digestive cancer. Psychiatry Clin Neurosci 2005;59(5):576-583. doi: https:// doi.org/10.1111/j.1440-1819.2005.01417x. PubMed PMID: 16194261.

49. Patsou ED, Alexias GD, Anagnostopoulos FG, Karamouzis MV. Effects of physical activity on depressive symptoms during breast cancer survivorship: a meta-analysis of randomised control trials. ESMO open. 2017;2(5):e000271. Epub 2017/12/21. doi: https://doi.org/10.1136/esmoopen-2017-000271. PubMed PMID: 29259819; PubMed Central PMCID: PMCPMC5729305.

50. Wu PH, Chen SW, Huang WT, Chang SC, Hsu MC. Effects of a psychoeducational intervention in patients with breast Cancer undergoing chemotherapy. J Nurs Res 2018. doi: https://doi.org/10.1097/jnr. 0000000000000252. PubMed PMID: 29360672.

51. Tabrizi FM, Radfar M, Taei Z. Effects of supportive-expressive discussion groups on loneliness, hope and quality of life in breast cancer survivors: a randomized control trial. Psycho-Oncology 2016;25(9):1057-1063. Epub 2016/06/16. doi: https://doi.org/10.1002/pon.4169. PubMed PMID: 27302306

52. Chen SF, Wang HH, Yang HY, Chung UL. Effect of Relaxation With Guided Imagery on The Physical and Psychological Symptoms of Breast Cancer Patients Undergoing Chemotherapy. Iranian Red Crescent Med J. 2015; 17(11):e31277. Epub 2016/01/07. doi: https://doi.org/10.5812/ircmj.31277. PubMed PMID: 26734485; PubMed Central PMCID: PMCPMC4698327.

\section{Ready to submit your research? Choose BMC and benefit from}

- fast, convenient online submission

- thorough peer review by experienced researchers in your field

- rapid publication on acceptance

- support for research data, including large and complex data types

- gold Open Access which fosters wider collaboration and increased citations

- maximum visibility for your research: over $100 \mathrm{M}$ website views per year

At BMC, research is always in progress.

Learn more biomedcentral.com/submissions 